\title{
LONGÉVITÉ DE L'INFORMATION NUMÉRIQUE
}

\author{
Les données que nous voulons garder \\ vont-elles s'effacer?
}

Rapport du

groupe PSN (pérennité des supports numériques)

commun à l'Académie des sciences

et à l'Académie des technologies

Membres du groupe :

Erich Spitz,

Académie des sciences et Académie des technologies, président

Jean-Charles Hourcade,

Académie des technologies

Franck Laloë,

LKB/ENS, rapporteur 
Conception de la couverture : Jérôme Lo Monaco

Maquette et mise en pages : Patrick Leleux PAO (Lisieux)

Imprimé en France

ISBN : 978-2-7598-0509-9

Tous droits de traduction, d'adaptation et de reproduction par tous procédés, réservés pour tous pays. La loi du 11 mars 1957 n'autorisant, aux termes des alinéas 2 et 3 de l'article 41, d'une part, que les «copies ou reproductions strictement réservées à l'usage privé du copiste et non destinées à une utilisation collective ", et d'autre part, que les analyses et les courtes citations dans un but d'exemple et d'illustration, «toute représentation intégrale, ou partielle, faite sans le consentement de l'auteur ou de ses ayants droit ou ayants cause est illicite» (alinéa $1^{\mathrm{er}}$ de l'article 40). Cette représentation ou reproduction, par quelque procédé que ce soit, constituerait donc une contrefaçon sanctionnée par les articles 425 et suivants du code pénal.

(c) EDP Sciences 2010 